\title{
The state of the medical geneticist workforce: Findings of the 2003 survey of American Board of Medical Genetics certified geneticists
}

Judith A. Cooksey, MD, MPH' ${ }^{1}$, Gaetano Forte, BA ${ }^{2}$, Judith Benkendorf, MS, CGC $C^{1,3}$, and Miriam G. Blitzer, PhD Key Words: genetics workforce, 2003 ABMG survey, medical geneticist, clinical laboratory geneticist, genetic services

Will the United States have sufficient numbers of qualified health professionals to provide for the future genetic health care and service needs of the population?

This is a difficult question to answer. Continued scientific discoveries and new clinical applications will fuel a growing interest and demand for genetic services. At the same time, the very definition of genetic services is evolving. Clinical genetics services are highly specialized and currently constitute a focused area of overall medical care and public health programs. Yet, genetics-related discoveries are forecast to be one of the most significant factors affecting health care over the next decades.

This summary presents key findings of a survey of medical geneticists that was one component of a 3-year national research project, Assessing Genetic Services and the Health Workforce. The goals of the overall project were to: (1) describe current models of providing clinical genetics services; (2) describe the roles of health professionals delivering services; (3) identify measures to monitor changes in demand for services; and (4) establish a framework and baseline description for ongoing and longitudinal studies of genetic services.

A written survey of all American Board of Medical Genetics (ABMG) certified medical geneticists was conducted in February 2003; it included 67 questions organized into six sections. A $55 \%$ response rate was obtained. No response rate difference (bias) was found using comparisons by geographic location (US Census regions and divisions), and year and type of initial ABMG certification. When using comparisons by respondents' degree type, response rates varied from $51 \%$ to $63 \%$, with a slight but statistically significant higher response rate for the PhD-only degree subgroup. We concluded from this analysis that the observed response rates showed variability but little evidence of bias. Thus, we present findings weighted for

\footnotetext{
From the ${ }^{1}$ Genetics Health Services Research Center, Department of Epidemiology and Preventive Medicine, University of Maryland School of Medicine, Baltimore, Maryland; ${ }^{2}$ Center for Health Workforce Studies, University at Albany, SUNY, School of Public Health, Albany, New York; ${ }^{3}$ American College of Medical Genetics, Bethesda, Maryland; and ${ }^{4}$ Department of Pediatrics, University of Maryland School of Medicine, Baltimore, Maryland.

Judith A. Cooksey, MD, MPH, Genetics Health Services Research Center, Department of Epidemiology and Preventive Medicine, University of Maryland School of Medicine, 660 West Redwood St., Baltimore, MD 21201.
}

DOI: 10.1097/01.GIM.0000172416.35285.9F the entire population (1377) of living, US resident, active medical geneticists certified before 2002. The 146 medical geneticists certified in 2002/2003 were not included in the survey, due to timing.

This commentary begins with a workforce summary that presents findings for the entire medical genetics workforce. Only limited subgroup analysis is presented (e.g., the geographic distribution of MD clinical geneticists, activities of clinical laboratory geneticists). This workforce summary approach allows one to describe the overall characteristics, professional practices, and aggregate contributions of the professional group.

\section{BACKGROUND}

Medical geneticists are medical specialists (MDs and PhDs) specifically trained to provide clinical genetics services. They work in direct patient care and diagnose, manage, and treat individuals with genetic conditions, counsel individuals and families at risk for genetic conditions, and serve as consultants to physicians and other health professionals. Geneticists also direct clinical laboratories, conduct basic biomedical and clinical research, educate health professionals and trainees, and serve as leaders and senior administrators of organizations involved with genetics-related research, education, patient care, laboratory medicine, and public health. Medical geneticists and others trained in clinical genetics are expected to help lead the integration of new genetic or genomic medicine knowledge and skills into clinical practice.

Medical genetics is one of the smallest of the 24 member boards of the American Board of Medical Specialties (ABMS). We estimate that there are approximately 1525 professionally active board-certified (American Board of Medical Genetics, ABMG) medical geneticists in the United States, representing $1025 \mathrm{MD}$ geneticists and about $500 \mathrm{PhD}$ geneticists (Cooksey, unpublished data, February, 2005). Medical geneticists made up $<0.4 \%$ of all physicians certified in the last 10 years (19932002). ${ }^{1}$ Medical genetics is also a relatively new specialty, with board certificates first issued in 1982; the ABMG became the newest member of the ABMS in 1991.2

The specialty of medical genetics includes five certification and training options. The MD clinical genetics specialization is 
only available to physicians and requires a minimum of 4 years of graduate medical education (GME) with at least two of these years in clinical genetics training. The ABMG reports that most geneticists also are certified in another medical specialty, most commonly in pediatrics (68\%), followed by obstetrics and gynecology $(13 \%)$, internal medicine $(10 \%)$, pathology $(4 \%)$, and psychiatry and neurology (3\%). ${ }^{3}$ The laboratory specializations require either an $\mathrm{MD}$ degree or a $\mathrm{PhD}$ in genetics or a related field and at least 2 years of clinical postdoctoral training in one of the three clinical genetics laboratory specialties. The $\mathrm{PhD}$ medical genetics certification exam will be offered for the last time in 2007.

\section{Supply and geographic distribution of MD clinical geneticists}

The national supply of active MD clinical geneticists, the group most likely to be involved with direct patient care, is approximately $3.5 \mathrm{MD}$ clinical geneticists per million people. ${ }^{1}$ The geographic distribution at the state level is varied and likely reflects locations of institutions that employ clinical geneticists such as academic medical centers, or metropolitan areas that have major medical referral centers or have large genomics research centers. Thus, a few states have a disproportionately high supply relative to their population, and many states have population-adjusted supplies that are quite low.

Across many regions of the country, notably the Midwest and South, and the Mountain division of the West region, the supply of MD clinical geneticists relative to population is small in all but a few states. Ten states stand out as having very few MD clinical geneticists and very low population-adjusted ratios; two states have no geneticists, five states have one geneticist, and two states have two geneticists. Another seven states have 2.0 or fewer geneticists per one million people. Based on state factors that could affect access (i.e., large rural state, small rural state without high supply states nearby, or an isolated state), we would consider each of these 17 states to have an inadequate supply of medical geneticists for their state population. This finding suggests the potential for geographic access barriers for residents in these areas.

\section{KEY FINDINGS FROM THE ABMG SURVEY}

\section{Demographic characteristics and education and training}

Geneticists are equally represented by women (50\% of geneticists) and men, although slightly fewer women are MD geneticists (45\%), compared to $\mathrm{PhD}$ geneticists (59\%). The average age of the geneticists in this study is 52 years. However, because the survey sample does not include the most recently certified group, this age distribution may be shifted slightly upwards. About $35 \%$ of geneticists are 55 years or older and $10 \%$ are 65 years or older. Few geneticists are members of racial or ethnic minority groups.

Geneticists are a highly educated and trained group. Although medical genetics is considered a primary medical specialty, most MDs have GME training and board certification in at least one other medical specialty in addition to medical ge- netics. Twenty-one percent of the MD geneticists also have a $\mathrm{PhD}$.

\section{Professional practice}

The primary work settings for medical geneticists are academic medical centers $(62 \%)$, hospitals (9\%), commercial laboratories $(9 \%)$, medical practices $(9 \%)$, and other settings $(10 \%)$. The median job tenure is 11 years; $23 \%$ of geneticists have been at their current employment setting for over 20 years. Geneticists are engaged in multiple professional activities, with their time in aggregate distributed across patient care (30\% of time), clinical laboratories $(22 \%)$, research $(21 \%)$, administration (10\%), education/teaching (8\%), and other activities (8\%). In aggregate, MD geneticists spend about $43 \%$ of their time in patient care and $\mathrm{PhD}$ geneticists spend about $50 \%$ of their time in clinical laboratories. When analyzed by individuals, $86 \%$ of MD geneticists report some patient care activity, although this does not necessarily equate with direct faceto-face care (see the following section).

Geneticists work on average 52 hours per week and 48 weeks per year. Few work less than full time ( $8 \%$ work fewer than 40 hours per week); many (35\%) work 60 or more hours per week. Over $90 \%$ of geneticists are employed and receive salary income; smaller numbers also have other professional income sources (e.g., consulting fees or honoraria, employer bonuses or incentives, patient fee revenues). The median net professional income (before taxes) in 2002 was $\$ 123,000$ for all geneticists; this was higher for MDs $(\$ 135,000)$ than $\mathrm{PhDs}$ $(\$ 101,000)$. About $20 \%$ of geneticists plan to retire in the next 10 years, about one quarter do not have retirement plans, and the remainder plan retirement after 10 years.

\section{Clinical genetics practice}

Historically, clinical genetics services have focused primarily on single gene disorders in infants and children, and assessing risks for genetic conditions in future offspring. Genetic services for adults largely had been limited to the uncommon single gene conditions that manifest symptoms in adulthood. At the aggregate analysis level, the patients seen in 2002-2003 continue to reflect these traditional referral patterns and also the geneticists' training.

About 70\% of all MD geneticists provide direct, face-to-face patient care. Patient care activities range from under $10 \%$ time to over $90 \%$ of individuals' work time. A majority $(73 \%)$ of these individuals were trained in pediatrics (called pediatric geneticists). The other MD geneticists were trained in obstetrics and gynecology or internal medicine, with fewer numbers trained in other specialties.

Children, from birth through adolescence, are the major patient group ( $76 \%$ of all new patients) seen by all geneticists. Also across all geneticists, reproductive genetics patients, including pregnant women, account for $11 \%$ of all new patient visits, and other adults for $13 \%$. Whereas infants/children/adolescents account for $81 \%$ of all new patient visits to pediatric geneticists, children only account for $5 \%$ of new patients seen by other geneticists. For the other geneticists, reproductive 
$(68 \%)$ and adult (27\%) genetics patients are the major patient groups. Some pediatric geneticists appear to practice as general geneticists, because in aggregate, adult and reproductive genetics patients account for $18 \%$ of pediatric geneticists' new patients. These data show practice delineation in the types of patients seen by subgroups of geneticists based on their nongenetics residency training.

Staffing levels and mix vary across the geneticists' primary clinical sites. About half of all patient care geneticists are in clinics with one or two geneticists, another $40 \%$ have three to five geneticists, and $12 \%$ have six or more geneticists. Most geneticists (85\%) work with genetic counselors in their clinics. Nurses and other professional staff are present in about half of the clinical sites.

Geneticists providing patient care, on average, spend only $50 \%$ of their professional time on patient care activities. They see an average of about 380 new patients per year and provide almost 300 follow-up patient visits per year. The new and follow-up patient visits are lengthy (85 and 43 minutes on average). The number of follow-up patient visits is small, reflecting the dominant practice pattern of geneticists as consultants, although some geneticists do provide ongoing management and treatment of patients.

The capacity of geneticists to see new patients was assessed through several questions, and findings show a mixed picture that suggests limited expansion capacity. About 70\% report their practice is nearly full or full, although $30 \%$ report they could accept many new patients. Slightly more than half of the geneticists could schedule a new patient within 3 weeks, and only $10 \%$ could not schedule a new patient for more than 3 months. About $60 \%$ report that the supply of geneticists in their community is not adequate for the demand for services. About $25 \%$ of geneticists perceive the supply and demand to be in balance, whereas 15\% perceive that the local supply exceeds demand.

Physicians, both primary care and other specialist physicians, are the major referral source of new patients. Approximately $70 \%$ of geneticists report that physicians are the referral source for many or all of their new patients, and another $28 \%$ report that physicians are a source for at least some of their referrals. About $20 \%$ of geneticists report many or all of their referrals are patient self-referrals, and almost all geneticists report at least some patient self-referrals. Genetic counselors are a referral source for some patients for $75 \%$ of geneticists. Managed care contracting and genetics laboratories are referral sources for some patients for about half of geneticists.

Almost $60 \%$ of geneticists report an increase in patient volume compared to 3 years ago, $12 \%$ report a decrease, and the remainder report no change. The complexity of patients has increased for $44 \%$ of geneticists, and the number of services provided per patient visit increased for about $30 \%$ of geneticists. Approximately $30 \%$ of geneticists reported increased use of genetic counselors. Most of the remaining respondents reported no change in these areas.

\section{Genetics clinical laboratory services}

About $38 \%$ of medical geneticists spend at least some work time in clinical genetics laboratories. In aggregate, these geneticists spend $50 \%$ of their overall professional time in clinical laboratory activities and the remainder in other professional activities. The largest numbers of laboratory medical geneticists work in cytogenetics laboratories (57\%), followed by molecular genetics laboratories (24\%), biochemical genetics laboratories (15\%), and screening laboratories (4\%). Test volume varies across laboratories, with the median number of tests from 1,500 to 3,500 tests per year, and a range of 15 tests to 95,000 tests.

Geneticists spend a majority of their clinical laboratory time in interpreting and reporting test results, followed by communication with other professionals, direct test-related activities, and case-related research. Staffing in laboratories includes geneticists and laboratory technicians or technologists in all labs. Genetic counselors and other professionals are employed by about $40 \%$ of labs.

When asked to compare laboratory practice to 3 years earlier, the following numbers of laboratory geneticists noted increases in the following: test volume (73\% of respondents); complexity of test interpretation (67\%); competition from other laboratories (62\%); and referrals from nongeneticist physicians (56\%). Whereas $76 \%$ of respondents reported increased operational costs, only $52 \%$ reported increased revenue from laboratory services, and $26 \%$ reported increased institutional financial support.

\section{Professional satisfaction and perspectives on access and quality of care}

Almost three quarters of all medical geneticists report being satisfied or very satisfied with their current practice or position, $12 \%$ are dissatisfied, and $14 \%$ are neutral. Most are satisfied or very satisfied with their relationships with geneticist colleagues and other health professionals, and with professional challenges. Lower satisfaction ratings were reported for income and earning potential, institutional support, and job-related stress.

When asked to assess policy-relevant issues, such as access to genetic services, almost half of medical geneticists rated access as only poor or fair, one third rated access as good, and less than one in five rated access as very good or excellent. About $75 \%$ of geneticists rated reimbursement for genetic services as poor or fair, and only $10 \%$ gave it a good or better rating ( $14 \%$ had no opinion).

\section{CONCLUSION}

\section{The medical genetics workforce situation is critical}

Based on findings from this survey and our comprehensive study of clinical genetics services, we believe that the situation facing the medical genetics workforce is critical and will probably become more serious in the near future. The medical genetics workforce, as currently constituted and directed, does 
not appear sufficient to meet expected patient care needs for clinical genetics services in the next 5 to 15 years. Listed below are several findings that support this conclusion.

\section{There is a serious mismatch between the expansion of knowledge and clinical applications in the field of medical genetics and the size of the medical genetics workforce.}

Among all areas of medicine, medical geneticists accounted for less than one-half of one percent of newly board-certified physicians in the past decade. At the same time, genomicsrelated knowledge is expanding rapidly in the laboratory arena and in preclinical applications across common medical conditions. Yet new entrants into the profession are declining!

Overall, only small numbers of medical geneticists have been trained and board certified over the last two decades. In addition, about 300 currently active medical geneticists plan to retire within the next 10 years. In recent years, the numbers of new geneticists have been falling. For MD clinical geneticists, there were 26 new diplomates per year over the last 6 years (1997 to 2002), compared to 42 new diplomates per year in the prior 12 years (1985 to 1996). ${ }^{4}$ For clinical laboratory geneticists, there were about 33 new diplomates per year over the last 6 years.

Although this information is not news for those within the profession, it is a striking and perplexing situation. For those considering future genetics workforce needs and planning for access to high quality clinical genetics patient care, this mismatch presents a serious problem and paradox. The obvious question is why has the profession not grown and attracted new entrants?

\section{Young physicians are not entering clinical genetics residencies. Competition among medical specialties for new trainees may grow as the nation faces another physician shortage.}

In 2004, there were $46 \mathrm{MD}$ clinical genetics residency programs across the country, with 193 approved positions; but there were only 82 physician trainees, or about 40 in each of the 2 years of training. ${ }^{4}$ This low fill rate for residency positions is partially influenced by the lack of funding for positions, although many other factors may contribute.

The number of genetics trainees is very small when one considers the number of US medical students that graduate each year $(18,500)^{5,6}$ or the first-year residency positions that fill across all residency programs (over 22,000). ${ }^{7}$ (The filled residency positions include international medical graduates as well as US graduates).

In an effort to address this situation, a Summit Meeting on the Training of Physicians in Medical Genetics was held in October $2004 .{ }^{4}$ The participants considered several options to recruit greater numbers of trainees to clinical genetics.

In addition, there is an emerging consensus within the physician workforce research community that the United States is on the brink of another extended physician shortage period. ${ }^{8-10}$ If this is true, it may make recruitment of young physi- cians into clinical genetics residencies even more difficult due to competition from other medical specialties.

\section{Many states and areas of the country have an inadequate supply of MD clinical geneticists to meet population demand for services.}

Based on our state level analysis, we determined that 17 states appear to have an inadequate supply of MD clinical geneticists to meet population needs. With more in-depth analysis, we believe that other states could be identified. Currently, substantial numbers of geneticists report their communities have an insufficient supply of clinical geneticists to meet patient demand for services. Almost half of geneticists rate access to genetic services as fair or poor. About $60 \%$ of geneticists have seen increases in patient volume over 3 years, and $70 \%$ rate their practice as full or nearly full. A full practice could reflect local high demand for a particular type of geneticist or simply any geneticist.

We believe that these data, although subject to only preliminary analysis, suggest that some areas of the country have an inadequate supply, or shortage, of geneticists.

\section{Practice specialization among geneticists, without expansion of the overall medical geneticist workforce, may create additional access problems for patient subgroups.}

The survey found that many practicing geneticists have a preference for, and in practice see, only certain categories of patients. As noted above, geneticists without pediatrics training report seeing few infants and children. Only some pediatrics-trained geneticists practice as general geneticists, that is, they see some number of adults or reproductive genetics patients. Possible explanations for this practice restriction could relate to the geneticists' personal interests and training, research areas, or other career or job choices.

However, other findings suggest that practice delineation may be due to the fast pace of genetics discovery that may herald an era of more specialized genetics practice. For example, many practicing geneticists interviewed as part of our overall study said they find it almost impossible to keep up with the knowledge explosion in their specific area of genetics practice, and that they cannot keep abreast of new advances much beyond their area(s) of interest. The tendency for professionals facing a situation of rapidly expanding knowledge is to narrow their scope of practice in order to maintain competency.

We think this practice delineation, or subspecialization among geneticists, may be a larger issue than generally recognized, and that it may have consequences for geneticist workforce planning and access to genetics care. This issue becomes particularly important if the number of new entrants to the profession remains small, and if there are increased numbers of geneticists that choose to narrow their genetics practice focus. If medical geneticists in particular geographic areas or among practice subgroups in relatively short supply further narrow their scope of practice, this may reduce overall access to genetic services in some communities and for certain subgroups of patients. This issue has not been systematically studied, but it 
should be examined to allow the profession to consider the implications.

There may be another emerging trend that further complicates the picture. Our overall project found that some medical specialists and subspecialists (such as neurologists and medical oncologists) are beginning to integrate genetic evaluations for specific conditions into their practice. These specialists may expand overall capacity for genetics evaluations for selected conditions, but their impact on meeting the overall demand for clinical genetic services may remain small over the next several years or longer. It will be difficult to assess this emerging practice model and the aggregate contributions of this model to overall genetics workforce planning.

\section{Medical geneticists will continue to carry out leadership roles in at least five critical genetics-related domains that impact health and society. The demands in each domain are expected to rise.}

Medical geneticists play many roles in advancing genetics for health and society. They currently distribute their time and expertise in clinical and human genetics across five large domains (patient care, clinical laboratory work, basic biomedical and clinical research, teaching, and administration). In many instances, there is only a small pool of nongeneticists that can fill these roles, and each domain is becoming increasingly complex and demanding.

Geneticists report increasing volume and complexity of patient care services and clinical laboratory genetic services are increasing in complexity, volume, and scale. Research opportunities and teaching responsibilities are also expected to increase. Many geneticists take on leadership roles with administrative responsibilities within their institutions or with national study groups or associations. They also fill service and policy-related roles (e.g., serving on state or national advisory and working groups developing policy papers or clinical guidelines, and assisting genetics-related advocacy groups).

\section{Implications}

The findings from this survey, our larger study, and other sources suggest the current situation for the medical geneticist workforce is critical. This situation merits high level and broad-based discussion, with serious review of all evidence, followed by appropriate actions. Leaders in medical genetics have recognized this problem for several years, and they have taken some steps to address it. They are now considering further options and actions.
Traditionally geneticists and genetic counselors have shared dialogue on many topics and this should continue with regard to critical genetics workforce issues. However, we believe that any serious dialogue on the future of genetic services and plans to assure access to services should also bring other key professional provider groups to the table. These include geneticssavvy medical specialists and primary care physicians, and advanced practice nurses in genetics. The American College of Medical Genetics is well positioned to take a leadership role in this important task.

\section{ACKNOWLEDGMENTS}

We acknowledge the generous support provided by a cooperative agreement (U78 HP 00016-01) with the Health Resources and Services Administration (HRSA) Bureau of Health Professions (BHPr) and Maternal Child Health Bureau, Genetics Services Branch, and the NIH National Human Genome Research Institute (NHGRI) Ethical, Legal, and Social Implications (ELSI) Program. We wish to acknowledge the special contributions to survey design by Ann C.M. Smith, MA, CGC, Beth A. Pletcher, MD, FAAP, and Edward Salsberg, MPP. Other members of the research team contributed to our overall understanding of genetic services, including Laura Mae Baldwin, MD, MPH, Daniel Beckett, MA, Patricia Flanagan, MA, Dale Halsey Lea, RN, MPH, CGC, APNG, Helen Travers, MS, CGC, Julie Mansour, MBA, Kyra Kissam, PhD, Mark Beaulieu, $\mathrm{PhD}$, Cynthia Gordon, $\mathrm{PhD}$, and Pamela Saunders, $\mathrm{PhD}$.

\section{References}

1. American Board of Medical Specialties (ABMS), The ABMS Annual Report and Reference Handbook, 2003 (Table 7, p 84) Available at: http://www.abms.org. Accessed April 28, 2005.

2. American Board of Medical Genetics (ABMG) web site. Available at: http://www. abmg.org. Accessed April 28, 2005.

3. American Board of Medical Genetics web site. 2004 Numbers of Certified Specialists in Genetics. Available at: http://www.abmg.org/genetics/abmg/stats-allyears.htm. Accessed April 28, 2005.

4. Korf BR, Feldman G, Weisner GL. Report of Banbury Summit meeting on training of physicians in medical genetics, October 20-22, 2004. Genet Med 2005;7:439-443.

5. Association of American Medical Colleges (AAMC). The AAMC Data Book: Statistical Information Related to Medical Schools and Teaching Hospitals. Washington DC: AAMC, 2005.

6. Singer AM. 2003 Annual Report on Osteopathic Medical Education. Chevy Chase, MD: Association of American Colleges of Osteopathic Medicine, 2004.

7. Graduate Medical Education [Appendix]. JAMA 2004;292:1103-1105.

8. Cooper RA. Weighing the evidence for expanding physician supply. Ann Intern Med 2004;141:705-714.

9. Cooper RA, Getzen TE, Laud P. Economic expansion is a major determinant of physician supply and utilization. Health Serv Res 2003;38:675-696.

10. Blumenthal D. New steam from an old cauldron: The physician-supply debate. N Engl J Med 2004;350:1780-1787. 